\title{
Feature
}

\section{From clergymen to computers-the advent of virtual palaeontology}

Palaeontology was established as a science in the Victorian era, yet has roots that stretch deeper into the recesses of history. More than 2000 years ago, the Greek philosopher Aristotle deduced that fossil sea shells were once living organisms, and around $500 \mathrm{AD}$ Xenophanes used fossils to argue that many areas of land must have previously been submarine. In 1027, the Persian scholar Avicenna suggested that organisms were fossilized by petrifying fluids; this theory was accepted by most natural philosophers up until the eighteenth century Enlightenment, and even beyond. The late 1700 s were notable for the work of Georges Cuvier who established the reality of extinction. This, coupled with advances in the recognition of faunal successions made by the canal engineer William Smith, laid the framework for the discipline that would become known as palaeontology. As the nineteenth century progressed, the scientific community became increasingly well organized. Most fossil workers were gentleman scientists and members of the clergy, who self-funded their studies in a new and exciting field. Many of the techniques used to study fossils today were developed during this 'classical' period. Perhaps the most fundamental of these is to expose a fossil by splitting the rock housing it, and then conduct investigations based upon the exposed surface (Fig. 1). This approach has served the science well in the last two centuries, having been pivotal to innumerable advances in our understanding of the history of life. Nevertheless, there are many cases where splitting a rock in this way results in incomplete data recovery; those where the fossils are not flattened, but are preserved in three-dimensions. Even the ephemeral soft-tissues of organisms are occasionally preserved in a three-dimensional state, for example in the Herefordshire, La Voulte Sûr Rhone and Orsten 'Fossil Lagerstätten' (sites of exceptional fossil preservation). These rare and precious deposits provide a wealth of information about the history of life on Earth, and are perhaps our most important resource in the quest to understand the palaeobiology of extinct organisms. With the aid of twenty-first century technology, we can now make the most of these opportunities through the field of 'virtual palaeontology'-computer-aided visualization of fossils.

While the study of three-dimensional fossils-in particular those with soft-tissue preservation-is very valuable, much of the form may be buried within the host rock and not visible. Thus extracting maximum data from such specimens can be problematic. Isolation methods can be used to recover three-dimensional morphological information by separating the matrix from the specimen. For instance, fossils can simply drop or be washed out of rocks, a process which may be imitated in the laboratory through the sieving of loosely consolidated sediments. Alternatively, chemical processes which dissolve the matrix but not the specimen can also be employed; this approach is commonly used in micropalaeontology. While ef- fective when applicable, such methods rely on an amenable matrix. They also rarely preserve associations in weakly articulated fossils, and can damage finer structures. The use of physical abrasive tools to separate the fossil from the matrix is another alternative, but this also risks damage to the specimen, and rarely allows entire specimens to be exposed. All of these approaches, of course, only provide access to the external form of the fossil; internal morphology remains hidden.

'Virtual palaeontology' sidesteps these problems. The techniques employed are generally tomographic; that is, they involve the imaging of serial sections through a fossil. The resulting two-dimensional sec-

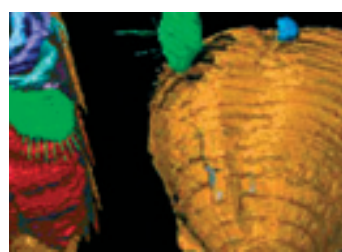


Fig. 1. A split nodule showing the fossil within, in this case a cockroachoid insect, $\times 2$.
Fig. 2. A fossil being serially ground. Here, a motorized arm is used to swipe the fossil and housing against a grinding plate.
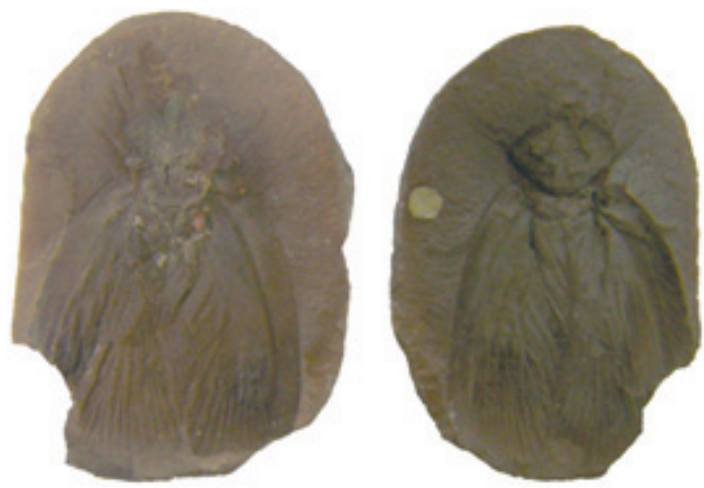

tion or slice images (tomograms) can either be studied directly, or used to create a three-dimensional model of the original fossil, often with the aid of a computer. These methods can be slow and/or destructive; however, they provide a means to study 'un-isolatable' three-dimensional fossils, as well as a way to peer into the internals of any such specimen. When combined with computer-based visualisation to create 'virtual fossils', datasets can be spun, zoomed, dissected and studied in a way no real fossil allows. This approach is not merely a solution for problematic material, but a powerful new set of techniques for revisiting any three-dimensionally preserved fossils. There are many approaches to obtaining slice-based (tomographic) datasets; three of the most important are outlined below.

\section{Physical-optical tomography}

The oldest method of recovering slice images - and the only approach available prior to the development of modern non-destructive scanning techniques-is physical-optical tomography. This was first used at the beginning of the twentieth century, and involves physical exposure and imaging of sections. Cross-sections of the specimen can be created through serial sawing; using a fine $(\sim 300 \mu \mathrm{m})$ saw and parallel cuts to expose surfaces millimetres apart. Because of the relatively high slice spacing, this approach is generally used to study larger organisms such as vertebrates, but has the advantage that it is only partially

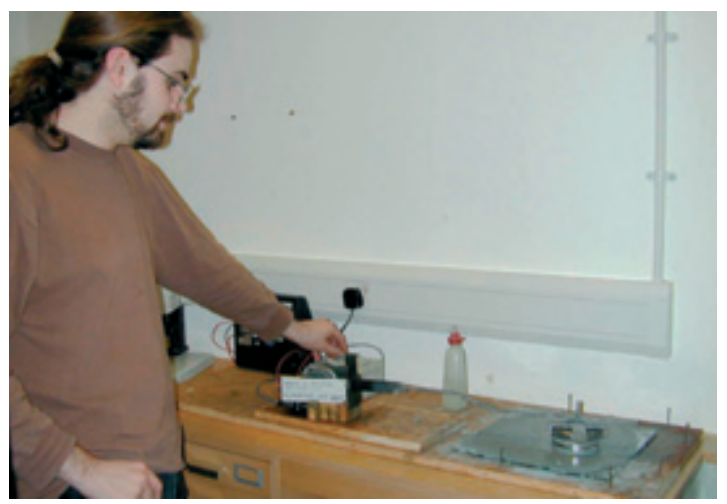

destructive, preserving material between saw cuts. A more commonly employed method is serial grinding (Fig. 2). This approach uses specialized grinding equipment to repeatedly remove small thicknesses from a specimen, with each newly exposed surface recorded. While totally destructive, the resulting surfaces are well polished and hence more easily visualised, and the approach is versatile in terms of scale, intervals down to $\sim 10 \mathrm{~m}$ having been achieved.

Exposed surfaces can be imaged in a number of ways. The pioneers of physical-optical tomography used traditional film photography, followed by the manual tracing of structures of interest. More recent work has employed digital photography, either microscope-mounted or direct, partly for its ease of use, and also to facilitate the production of computerized visualizations. Other means of data capture exist, most notably acetate peels. These have been historically popular as they provide physical records of exposed surfaces, and can be combined with staining to enhance contrast. However the inevitable presence of wrinkles, tears, contrast differences and bubbles in acetate-peel datasets renders digital reconstruction difficult.

High-fidelity visualizations require high-resolution datasets (good quality images of closely-spaced slices), consistent spacing, scale, brightness and contrast, and parallel slices. 'Registration' of the dataset prior to reconstruction is also required; each slice-image has to be manually rotated and shifted to ensure it is correctly aligned with the next. Thus physical-optical tomography is a time-consuming business, yet one capable of impressive results. An excellent example of recent work which has necessitated the use of this approach is the study of the "Herefordshire Lagerstätte'. This Silurian deposit preserves a diverse range of small invertebrates from the British Wenlock Series, laid down 425 million years ago (Ma). At this time, primitive members of most invertebrate phyla were still present, and these are preserved in extremely fine detail as external moulds in early diagenetic carbonate concretions. Following burial in a fine-grained volcanic ash, the organisms' tissues decayed entirely, leaving voids that were later infilled with calcite. The deposit preserved three-dimensional soft-parts in great detail, but because the calcite fossils are hosted in calcite nodules the fauna is very difficult to investigate with either isolation or scanning techniques. To recover the maximum amount of data from these fossils, physical-optical tomography has been employed, using serial grinding and digital photography. This approach has been used to create virtual models of a slew of important extinct taxa, including brachiopods, chelicerates, crustaceans, molluscs, and more besides (Fig. 3). Without tomography and virtual palaeontology this data would have remained locked up, its value largely unsuspected. 


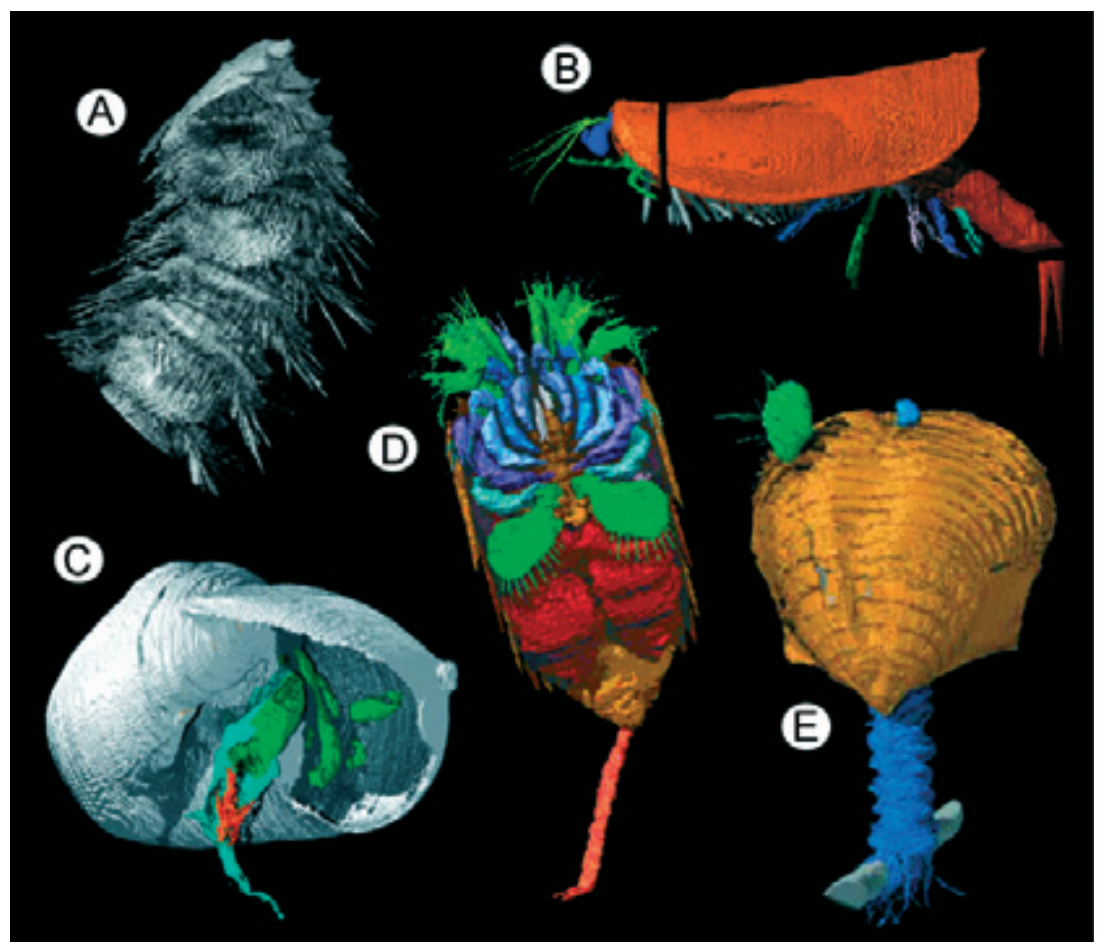

Fig. 3. Computer

reconstructions of fossils from the Silurian (Wenlock) Herefordshire Lagerstätte produced using physical-optical tomography. A. The mollusc Acaenoplax, $\times 10$ (from Sutton et al. 2001). B. The crustacean, Cinerocaris $\times 4$ (from Briggs et al. 2004). C. The gastropod Platyceras? $\times 11$ (from Sutton et al. 2006). D. The chelicerate Offacolus, $\times 9$ (from Sutton et al. 2002). E. The brachiopod Bethia, $\times 5$ (from Sutton et al. 2005). cus through a number of different planes, recording
Through these new techniques, the fossils are instead released in all their glory.

\section{Optical tomography}

Another method of generating tomograms is optical tomography; this technique is only applicable to fossils preserved within translucent materials like chert and amber. In such cases it is possible to use confocal laser scanning microscopy (a technique which allows single-plane focus) or conventional microscopy to foeach to build up a series of slice images. The resulting tomographic dataset can then be used to create a three-dimensional model. Optical tomography has recently been used to visualize fossils from the Rhynie Chert, Scotland. This translucent rock is Early Devonian (Pragian, $410 \mathrm{Ma}$ ) in age and preserves both a freshwater and terrestrial fauna. The represented community is thought to have lived in a hot springs environment, in and around a series of pools. The plants and animals were inundated by hot, silica-

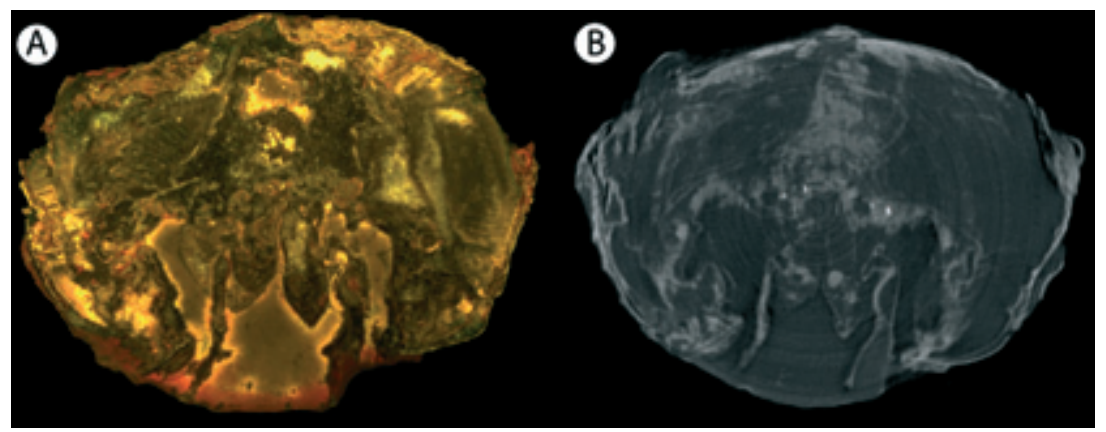

rich waters, trapping them within sinters (chemical crusts), which eventually became chert. This is a very important site, preserving life in exceptional detail very shortly after its emergence onto land. Optical tomography has been used to model the breathing apparatus of early arachnids from Rhynie, demonstrating similarities to modern terrestrial arachnids down to the microstructures of the lung, and once again demonstrating the efficacy of the 'virtual palaeontology' approach.

\section{X-ray computed tomography}

X-ray computed tomography $(\mathrm{CT})$ is a scanning technology which is used to acquire interior information for objects rapidly and non-destructively and, hence, is ideal for imaging irreplaceable fossil specimens. It was originally developed as a clinical diagnostic tool; however, during the $1980 \mathrm{~s}$, CT was co-opted for research in many other fields, including engineering, materials science, geology and palaeontology. Medical scanners are required to utilize relatively lowenergy X-rays and short exposure times to minimize patient radiation dosage, but non-medical CT has no such restrictions; such systems can be optimized to penetrate dense materials (such as rock) and to generate high-resolution datasets. In most CT scanners, Xrays are passed through a rotating sample; the degree to which the signal is attenuated (absorbed or scattered) is digitally recorded and used to compute virtual slice-images (tomograms) that map the variation of X-ray attenuation within the specimen (Fig. 4). $\mathrm{X}$-ray attenuation is roughly equivalent to density, so these tomograms allows distinct mineral phases to be differentiated-for example, distinguishing the fossil and the matrix - as long as they attenuate Xrays to a different extent. In palaeontological studies, medical CT scanners have been useful for imaging relatively large vertebrate fossils, but the miniaturized version of CT, X-ray microtomography, is the optimal technique for studying smaller vertebrate, plant and invertebrate fossils.

A novel and powerful variant of X-ray microtomography is synchrotron radiation X-ray tomographic microscopy, which uses a particle accelerator (the synchrotron) to generate extremely bright and monochromatic X-rays. In combination with the very highresolution X-ray detectors on synchrotron beamlines, this yields exceptionally high-quality tomographic data. This technique was applied to microscopic fossilized embryos from the Cambrian (542-488 Ma)

Fig. 4. Two tomograms (slice images) of the thylacocephalan arthropod Dollocaris. A. Physical-optical tomogram based on a digital photograph of a serially ground fossil, $\times 3$. B. CT tomogram, $\times 3$ 
of China and Siberia. These fossils are preserved by authigenic mineralization in phosphorites; they are too small (less than one millimetre in size) to study using CT or even conventional X-ray microtomography, necessitating the use of a synchrotron light source. Virtual reconstructions of the fossils were produced to decipher the anatomy of these enigmatic organisms (Fig. 5). This study identified various developmental stages in the fossil embryos and provided important phylogenetic information, confirming that some of the specimens represent the embryos of worm-like scalidophorans. Synchrotron sources are clearly invaluable investigative tools for palaeontologists that will become increasingly accessible in the future.

\section{Visualizing data}

It is informative to study tomographic datasets directly (i.e. as a set of slice-images); this approach has long been utilized in a number of palaeontological disciplines but is unsatisfactory for many reasons, not least being the simple difficulty of mentally visualizing structures from slices. Datasets are far more informative when studied as three-dimensional visualizations. Before the advent of computers capable of creating such reconstructions, all models were, by necessity, prepared physically; for example, layered wax cut-outs based upon the recovered tomograms were stuck together into fragile models. Such an approach is laborious, and makes manipulations, dissections and maintaining articulations difficult. With ever-increasing computer power, the last two decades have seen 'virtual palaeontology'-the use of computers to create digital visualizations - replace physical modelling. Such visualizations can be manipulated at will, dissected, and made locally translucent. Different structures can be individually coloured to aid interpretation, and there is no danger of fragile or poorly attached structures falling off. Unlike physical models, virtual models are also easy to copy and disseminate. If a more solid model is required they can be viewed in stereo using 3D glasses, or even used as the basis for a physical model through the use of rapid-prototyping technology ('3-D printing'). Virtual fossils provide a powerful new approach to working with palaeontological material; while developed as a solution to the problems posed by difficult specimens, in some ways they are now proving more productive to study than real fossils, and hence are likely to find very broad applications within palaeontology.

\section{Conclusions}

Tomographic virtual palaeontology entails the visualization of three-dimensionally preserved fossils from slice-image datasets. It is often the only practical approach for studying internal structures, which can be

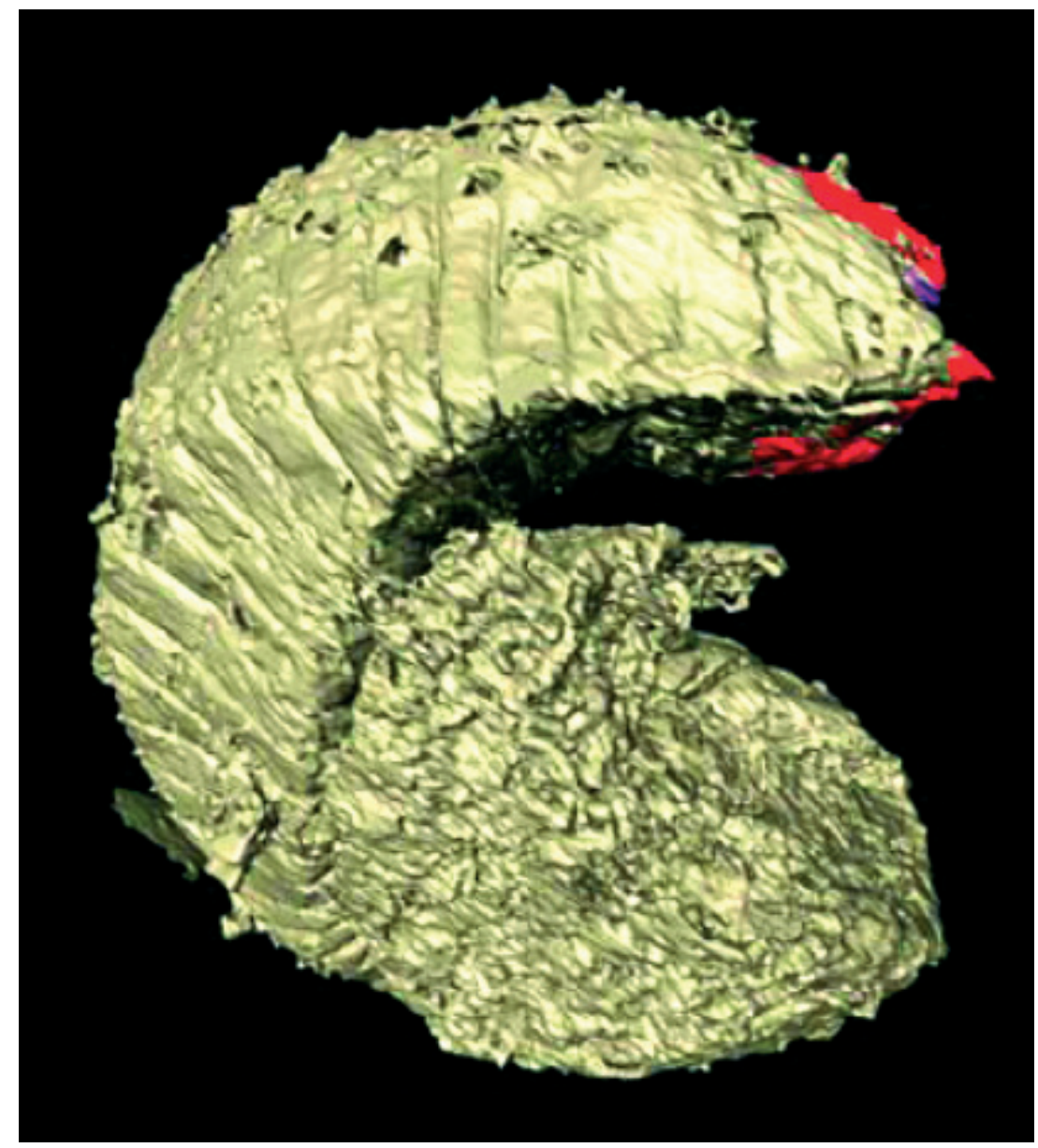

critical to a full understanding of the biology of fossil organisms. Furthermore, it provides a powerful new means of investigating external morphology; in some cases it is the only available approach. In the past, tomographic techniques, while widely used, have been hampered by their destructive nature and by the inadequacies of physical modelling approaches. The advent of powerful computers has now replaced physical models with far more satisfactory interactive digital visualizations, which are effective tools for research. While destructive physical-optical tomography remains the only option for some fossils-albeit with high-resolution digital photography to extract maximal data-others can now be investigated with non-destructive approaches such as optical tomography and scanning technologies. Optical tomography is limited to translucent materials, but scanning methods-primarily X-ray microtomography-are applicable to most fossils, and can provide highresolution models without the need to destroy the specimen. For more difficult specimens - those which are very small or too dense to allow the penetration of conventional X-rays-synchrotron scanning can be an effective alternative. Used in conjunction with traditional techniques, this powerful new way of working presents opportunities unimaginable to the
Fig. 5. Computer reconstruction of a fossilized embryo from the Upper Cambrian of Southern China, $\times 180$ (from Donoghue et al. 2006). 
gentleman scientists that founded palaeontology. The study of exceptionally preserved three-dimensional fossils-our most precious resource in the drive to understand the history of life on Earth-will never be the same again.

\section{Acknowledgements}

We thank D.E.G. Briggs, David J. Siveter, Derek J. Siveter and P.C.J. Donoghue for their permission to reproduce images.

\section{Suggestions for further reading}

Briggs, D.E.G., Siveter, D.J., Siveter, D.J. \& Sutton, M.D. 2008. Virtual fossils from 425 million-yearold volcanic ash. Scientific American, v.96, pp.474481.

Briggs, D.E.G., Sutton, M.D., Siveter, D.J. \& Siveter, D.J. 2004. A new phyllocarid (Crustacea: Malacostraca) from the Silurian Fossil-Lagerstätte of Herefordshire, UK. Proceedings of the Royal Society B, v.271, pp.131-138.

Donoghue, P.C.J., Bengtson, S., Dong, X., Gostling, N.J., Huldtgren, T., Cunningham, J.A., Yin, C., Yue, Z., Peng, F. \& Stampanoni, M. 2006. Syn- chrotron X-ray tomographic microscopy of fossil embryos. Nature, v.442, pp.680-683.

Kamenz, C., Dunlop, J.A., Scholtz, G., Kerp, H. \& Hass, H. 2008. Microanatomy of Early Devonian book lungs. Biology Letters, v.4, pp.212-215.

Sutton, M.D. 2008. Tomographic techniques for the study of exceptionally preserved fossils. Proceedings of the Royal Society B, v.275, pp.1587-1593.

Sutton, M.D., Briggs, D.E.G., Siveter, D.J. \& Siveter, D.J. 2001. An exceptionally preserved vermiform mollusc from the Silurian of England. Nature, v.410, pp.461-463.

Sutton, M.D., Briggs, D.E.G., Siveter, D.J., Siveter, D.J. \& Orr, P.J. 2002. The arthropod Offacolus kingi (Chelicerata) from the Silurian of Herefordshire, England: computer based morphological reconstructions and phylogenetic affinities. Proceedings of the Royal Society B, v.269, pp.1195-1203.

Sutton, M.D., Briggs, D.E.G., Siveter, D.J. \& Siveter, D.J. 2005. Silurian brachiopods with soft-tissue preservation. Nature, v.436, pp.1013-1015.

Sutton, M.D., Briggs, D.E.G., Siveter, D.J. \& Siveter, D.J. 2006. Fossilized soft tissues in a Silurian platyceratid gastropod. Proceedings of the Royal Society B, v.273, pp.1039-1044. 